\title{
Evaluación de la colonización de micorrizas arbusculares en pasto Bothriochloa pertusa (L) A. Camus
}

\author{
Colonization of arbuscular mycorrhizae in Bothriochloa pertusa \\ (L) A. Camus pasture
}

\begin{abstract}
Alexander Pérez C, ${ }^{1 *}$ Ph.D, Melba Vertel M, ${ }^{2}$ M.Sc.
${ }^{1}$ Universidad de Sucre, Facultad de Ciencias Agropecuarias. Campus universitario Puerta Roja, Sincelejo, Colombia. ${ }^{2}$ Universidad de Sucre, Facultad de Educación y Ciencias. *Correspondencia: alexander. perez@unisucre.edu.co
\end{abstract}

Recibido: Noviembre 28 de 2009; Aceptado: Julio 18 de 2010.

\section{RESUMEN}

Objetivo. Evaluar la colonización in situ de micorrizas arbusculares en raíces del pasto colosuana en función de factores físico-químicos de suelo, en fincas ganaderas de cuatro zonas agroecológicas, de la subregión fisiográfica de Sabanas. Materiales y métodos. La determinación del porcentaje de colonización fue realizada en raíces coloreadas. Para establecer diferencias entre porcentajes promedios de colonización entre zonas, se realizó un diseño completamente al azar. Para determinar diferencias entre zonas se utilizó la prueba de Tukey al 5\% de significancia. Las variables físico-químicas y los porcentajes de colonización en las fincas muestreadas fueron caracterizados por la técnica de análisis en componentes principales y clasificadas las fincas por análisis de Cluster aglomerativo. Resultados. El ANOVA mostró diferencias significativas ( $p$-value $=0.02359$ ) entre los porcentajes de colonización promedio entre zonas. La prueba de Tukey señaló que las zonas 2 y 4 presentaron los menores porcentajes de colonización y fueron estadísticamente iguales, mientras que las zonas 1 y 3 tuvieron los máximos valores de colonización. El análisis en componentes principales demostró altos porcentajes de colonización de HMA en las fincas que presentaron suelos con contenidos moderados de Fosforo y Nitrógeno; bajos contenidos de Sodio y pH moderadamente alcalinos. El Clúster aglomerativo mostró 5 grupos de fincas relacionadas con porcentajes de colonización en forma descendente cuando estuvo presente textura arcillosa a franco arenosa. Conclusiones. Este es el primer trabajo a nivel regional y nacional sobre el efecto de parámetros físicos-químicos del suelo sobre la colonización de micorrizas arbusculares en raíces del pasto colosuana.

Palabras clave: Micorrizas, colonización, pasto, análisis cluster. 


\section{ABSTRACT}

Objective. Evaluate in situ the colonization of arbuscular micorrhizae, in grass roots colosuana in function of the physical and chemical characteristics of the soil, in cattle farms of four agroecological areas of the savannah physiographical sub region. Materials and methods. The determination of the colonization percentage was done in colored roots. In order to establish the differences between average colonization percentages between areas, a completely random design was used. To determine the differences between areas, the Tukey test was used to the nearest $5 \%$. The physical and chemical variables and colonization percentages in the tested farms were characterized through the main component analysis technique, and the farms were classified through agglomerative cluster analysis. Results. The ANOVA showed significant differences $(p$-value=0.02359) between the colonization average percentages between areas. The Tukey test revealed that areas 2 and 4 showed the least colonization percentages and are statistically the same, while areas 1 and 3 had the maximum colonization values. The main component analysis showed high colonization percentages of HMA in farms with moderate phosphorous and Nitrogen contents; low sodium and moderately alkaline $\mathrm{pH}$. The agglomerative cluster showed 5 groups of farms related to decreasing colonization percentages in the presence of clay to sandy-loam structure.

Conclusions. This is the first regional and national level on the effect of physical-chemical parameters of soil on arbuscular mycorrhizal colonization in Colosuana grass roots.

Key words: Micorrhizae, colonization, grass, analysis Cluster.

\section{INTRODUCCIÓN}

En los últimos años ha despertado interés las interacciones entre las plantas y los hongos formadores de micorrizas arbusculares (HMA). Los HMA son microorganismos del suelo que forman simbiosis con mas del $95 \%$ de las plantas terrestres $(1,2)$, formando arbúsculos, vesículas (en algunas especies) e hifas, dentro de las células corticales de las plantas que colonizan (1). Tanto los hongos como las plantas tienen distribución universal, presentándose de esta manera ecotipos adaptados a condiciones diversas y extremas. Es de señalar que las plantas y las micorrizas tienen un origen común (2). Las HMA están ampliamente distribuidas en condiciones naturales, se encuentran en todos los continentes, excepto en la Antártida; se dan en todos los suelos, incluyendo los de minas abandonadas, suelos agrícolas, suelos de pantanos y en hábitat acuáticos $(3,4)$.

Los hongos formadores de micorrizas arbusculares son uno de los componentes de los ecosistemas naturales, representan entre el 5 a $50 \%$ de la biomasa de los microbios del suelo y son considerados como una comunidad biológica diversa y activa esencial para incrementar la sostenibilidad de los agroecosistemas. Las asociaciones con hongos micorrízicos representan las simbiosis de mayor relevancia en los sistemas agroecológicos. Los suelos poseen naturalmente una diversidad de especies de micorrizas, que pueden colonizar las raíces de la mayoría de las plantas cultivadas, independientemente de las condiciones ambientales, mejorando así el suministro de nutrientes, crecimiento y producción de las plantas hospederas especialmente en condiciones de nutrientes deficientes $(5,6)$.

Se estima que el pasto colosuana o kikuyina (Bothriochloa pertusa) (L). A. Camus, alcanza un total de 274.005 ha, distribuidas en 19 municipios del departamento de Sucre. El municipio de Corozal cuenta con la mayor área sembrada con esta especie de pasto en la región (32.223 ha). En el departamento de Sucre, la ganadería de doble propósito representa un $84.9 \%$ de su territorio (7). Una de las limitantes en la productividad animal es la escasez o falta total de forraje durante la época seca debido a la estacionalidad de las 
lluvias. Esta escasez es afectada cuando los suelos presentan diferentes grados de compactación, problemas erosivos, bajos niveles de fertilidad, la falta de abonamientos y el pastoreo extensivo, lo que genera la degradación de las praderas.

Existen escasas investigaciones a nivel mundial y nacional que demuestren integración entre los parámetros físicos, químicos y biológicos con relación a la calidad del ecosistema suelo. Las informaciones que existen son pocos conclusivas y de difícil interpretación, dada la gran complejidad del sistema en estudio en donde se evalúan varias características, generando un número mayor de datos, lo que proporcionalmente dificulta su interpretación. Una estrategia para evaluar el conjunto de indicadores biológicos asociados a los ecosistemas comúnmente sugeridos es el análisis a través de herramientas de bioestadística (análisis multivariados) que permita la conversión e interpretación de un conjunto de variables con una alta correspondencia entre ciertos indicadores y un componente, resultando en un alto peso absoluto del indicador en un componente determinado (8).

Se ha evidenciado la importancia que tiene la colonización de los HMA con las raíces de las plantas, en relación con el mejoramiento de las condiciones físico-químicas del suelo; la estimulación del crecimiento e incremento de la calidad nutricional de las especies vegetales, convirtiéndolas en más tolerantes a condiciones adversas tanto abióticas como bióticas (9). Sin embargo existe escasa información in situ, del efecto de estos hongos con las plantas; debido a que in vivo es difícil de evaluar la relación entre las variables ambientales y la colonización de micorrizas arbusculares sobre las plantas.

A partir de la anterior situación, surge la necesidad de evaluar in situ la colonización de raíces con hongos formadores de micorrizas arbusculares en relación a parámetros físicos y químicos del suelo. Estos hechos justifican la profundización de estudios conducentes a determinar el efecto de esos componentes biológicos sobre agroecosistemas de pasturas. El presente trabajo tuvo como objetivo evaluar el efecto de la colonización in situ de micorrizas arbusculares nativas, asociadas a raíces de la especie de pasto Bothriochloa pertusa ( $L$ ) A. Camus, en función de parámetros físicoquímicos de suelo de fincas ganaderas de cuatro zonas agroecológicas, de la subregión fisiográfica de Sabanas.

\section{MATERIALES Y MÉTODOS}

Sitio de estudio. Se realizó en el municipio de Corozal-Sucre (Colombia), ubicado en la región fisiográfica de Sabanas que constituye el declive general de los montes de María hacia la depresión del bajo Cauca y San Jorge; a los $8^{\circ} 55^{\prime \prime}$ y $9019^{\prime \prime}$ de latitud Norte, y entre $75^{\circ} 25^{\prime \prime}$ y $74^{\circ} 42^{\prime \prime}$ al Este del meridiano de Greenwich; con temperatura promedio anual de $28^{\circ} \mathrm{C}$, pluviosidad de $1105 \mathrm{~mm}$ anuales y humedad relativa del $80 \%$, a una altura entre 174 a 200 msnm.

Selección de fincas. Se tomaron 48 fincas ganaderas del municipio de Corozal (Sucre) con pastura predominante de Bothriochloa pertusa $(L) A$. distribuidas en cuatro zonas agrologicas según la clasificación de tierras por su capacidad de uso y manejo $(10,11)$ : zona 1 (III sc - IV hs), zona 2 (VIIsc - IV esc), zona 3 (VI sc - VI esc) y zona 4 (VII sc - VII esc).

Recolección de muestras. Un tubo plastico de PVC de $3.8 \mathrm{~cm}$ de diámetro y $25 \mathrm{~cm}$ de longitud, fue usado para tomar las muestras a una profundidad entre 0-20 $\mathrm{cm}$, introduciendo, rotando y extrayendo el cilindro con la muestra (suelo y raíces). En cada finca se tomaron entre 15-20 muestras, estas se homogenizaron por finca para conformar una con un peso de 2000 gramos. Dos submuestras (1000 g cada una) fueron empleadas para determinar el porcentaje de colonización en raíces con hongos formadores de micorrizas arbusculares, y una submuestra de 1000 $\mathrm{g}$ para las determinación de parámetros físico-químicas del suelo (Sodio "Na", Fósforo "P", Nitrógeno "N", pH, Capacidad de Intercambio Catiónico "CIC", Calcio "Ca", Magnesio "Mg", Aluminio "Al" y Potasio "K", Materia orgánica "MO"). 
Las muestras etiquetadas en bolsas plásticas se llevaron al laboratorio y se conservaron a $4^{\circ} \mathrm{C}$ hasta su posterior análisis.

Determinación de HMA. El porcentaje de colonización de HMA en raíces de Bothriochloa pertusa $(L) A$. fue determinado mediante técnica de coloración de raíces. Las raíces coloreadas fueron colocadas sobre láminas cubiertas con laminillas, para su observación con objetivo 40X. Para estimar el porcentaje de colonización en raíces se contaron 100 campos ordenadamente por muestra. En cada muestra se observó la presencia de campos negativos (sin presencia de estructuras colonizantes) y positivos (con presencia de estructuras colonizantes). En los campos positivos se tuvo en cuenta el tipo de estructuras (arbusculos, vesículas, hifas y esporas) presente dentro de cada raíz (12).

Análisis estadístico. Se utilizó un diseño completamente al azar empleando las zonas agroecológicas como tratamientos, y repeticiones diferentes por tratamiento (fincas ganaderas muestreadas). Los datos obtenidos para todas las variables cuantificadas se sometieron a la prueba de Shapiro-Wilk para verificar su normalidad y el test de Bartlett para verificar la homogeneidad de varianzas. Se aplicó análisis de varianza (ANOVA) y prueba de comparación de tratamientos Tukey al nivel del $5 \%$ de significancia para verificar el efecto de los tratamientos sobre el porcentaje de colonización en raíces de HMA (13). No se obtuvo normalidad de las variables Nitrógeno, Fósforo, Sodio, Potasio, se realizó análisis de varianza (ANOVA) por el método no paramétrico de Kruskal-Wallis y separación de medias por rangos por el procedimiento de Tukey.

Las relaciones estadísticas entre variables físico-químicas y colonización en raíces de HMA sobre las fincas muestreadas fueron determinadas mediante el método multivariado análisis en componentes principales (ACP) (14). La clasificación de las fincas muestreadas teniendo en cuenta la información recolectada en el ACP fue realizada con el análisis de cluster aglomerativo de distancias euclidianas ligado al método de Ward $(15,16)$. Todos los datos numéricos fueron analizados en el programa estadístico R (17).

\section{RESULTADOS}

Teniendo en cuenta los resultados del análisis descriptivo (media \pm desviación estándar) y ANAVA para parámetros físicosquímicos en fincas ganaderas (Tabla 1), muestran valores de $\mathrm{pH}$ que oscilan de fuertemente ácidos a fuertemente alcalinos,

Tabla 1. Estadística descriptiva numérica (Media \pm desviación estándar), análisis de varianza y prueba de Tukey de parámetros químicos de suelos ganaderos por zonas agroecológicas.

\begin{tabular}{|c|c|c|c|c|c|}
\hline \multicolumn{6}{|c|}{ Zonas agroecológicas } \\
\hline Variables & Zona 1 & Zona 2 & Zona 3 & Zona 4 & ANOVA \\
\hline $\mathrm{pH}$ & $6.78 \pm 0.28 a$ & $7.52 \pm 0.16 a$ & $7.52 \pm 0.14 a$ & $5.44 \pm 0.20 \mathrm{~b}$ & $\mathrm{p}$-value $=2-08 * * *$ \\
\hline MO & $1.33 \pm 0.84 \mathrm{a}$ & $1.36 \pm 0.78 \mathrm{a}$ & $1.72 \pm 0.65 a$ & $1.23 \pm 0.89 a$ & $\mathrm{p}$-value $=0.3754^{\mathrm{NE}}$ \\
\hline $\mathrm{N}$ & $0.008 \pm 0.012 a$ & $0.0012 \pm 0.0008 a$ & $0.0017 \pm 0.00065 a$ & $0.0012 \pm 0.0008 \mathrm{a}$ & $\mathrm{p}$-value $=0.2840^{\mathrm{Ns}}$ \\
\hline$P$ & $7.70 \pm 6.85 a$ & $11.71 \pm 6.71 \mathrm{ab}$ & $6.62 \pm 2.56 \mathrm{bc}$ & $3.11 \pm 3.80 \mathrm{c}$ & $\mathrm{p}$-value $=0.0017 * * *$ \\
\hline $\mathrm{CIC}$ & $24.42 \pm 5.91 \mathrm{a}$ & $31.16 \pm 6.0 \mathrm{a}$ & $24.18 \pm 7.08 \mathrm{a}$ & $22.13 \pm 4.92 \mathrm{a}$ & $\mathrm{p}$-value $=0.2373^{\mathrm{Ns}}$ \\
\hline $\mathrm{Na}$ & $1.51 \pm 0.34 \mathrm{a}$ & $1.23 \pm 0.24 \mathrm{a}$ & $1.40 \pm 0.96 \mathrm{a}$ & $0.50 \pm 0.45 b$ & $\mathrm{p}$-value $=0.0004 * * *$ \\
\hline $\mathrm{K}$ & $0.17 \pm 0.10 \mathrm{a}$ & $0.23 \pm 0.16 a$ & $0.20 \pm 0.14 a$ & $0.51 \pm 0.51 a$ & $\mathrm{p}$-value $=0.3581^{\mathrm{Ns}}$ \\
\hline $\mathrm{Ca}$ & $5.82 \pm 2.75 a$ & $8.18 \pm 2.26 \mathrm{a}$ & $8.47 \pm 6.71 \mathrm{a}$ & $11.32 \pm 3.12 \mathrm{a}$ & $\mathrm{p}$-value $=0.5771^{\mathrm{N} 5}$ \\
\hline $\mathrm{Mg}$ & $3.95 \pm 1.98 \mathrm{a}$ & $3.84 \pm 1.15 \mathrm{a}$ & $3.76 \pm 1.81 \mathrm{a}$ & $6.47 \pm 5.75 a$ & $\mathrm{p}$-value $=0.6772^{\mathrm{Ns}}$ \\
\hline$\%$ colonización & $59.43 \pm 4.72 a$ & $40.12 \pm 4.67 \mathrm{ab}$ & $49.12 \pm 2.46 b$ & $40.58 \pm 2.70 b$ & $p$-value $=0.0235 *$ \\
\hline
\end{tabular}

Media \pm desviación estándar de las variables evaluadas para cada zona agroecológica. *Diferencias significativas a $p<0.05$, NS: No hay diferencias significativas al $5 \%$ Letras iguales no hay diferencias significativas entre zonas para cada variable 
contenidos medios a bajos de Fósforo, Materia Orgánica, Nitrógeno y Sodio, y valores altos a medios de Calcio, Magnesio y Potasio.

Los resultados de los análisis de varianza (Tabla 1) para parámetros químicos del suelo en zonas agroecológicas, señalan que hubo diferencias significativas entre los valores de $\mathrm{pH}$, Fosforo y Sodio del suelo en las diferentes zonas agroecológicas analizadas con relación a los otros parámetros químicos evaluados. No se obtuvo normalidad de las variables Nitrógeno, Fósforo, Sodio, Potasio, se realizó análisis de varianza (ANOVA) por el método no paramétrico de Kruskal-Wallis y separación de medias por rangos por el procedimiento de Tukey.

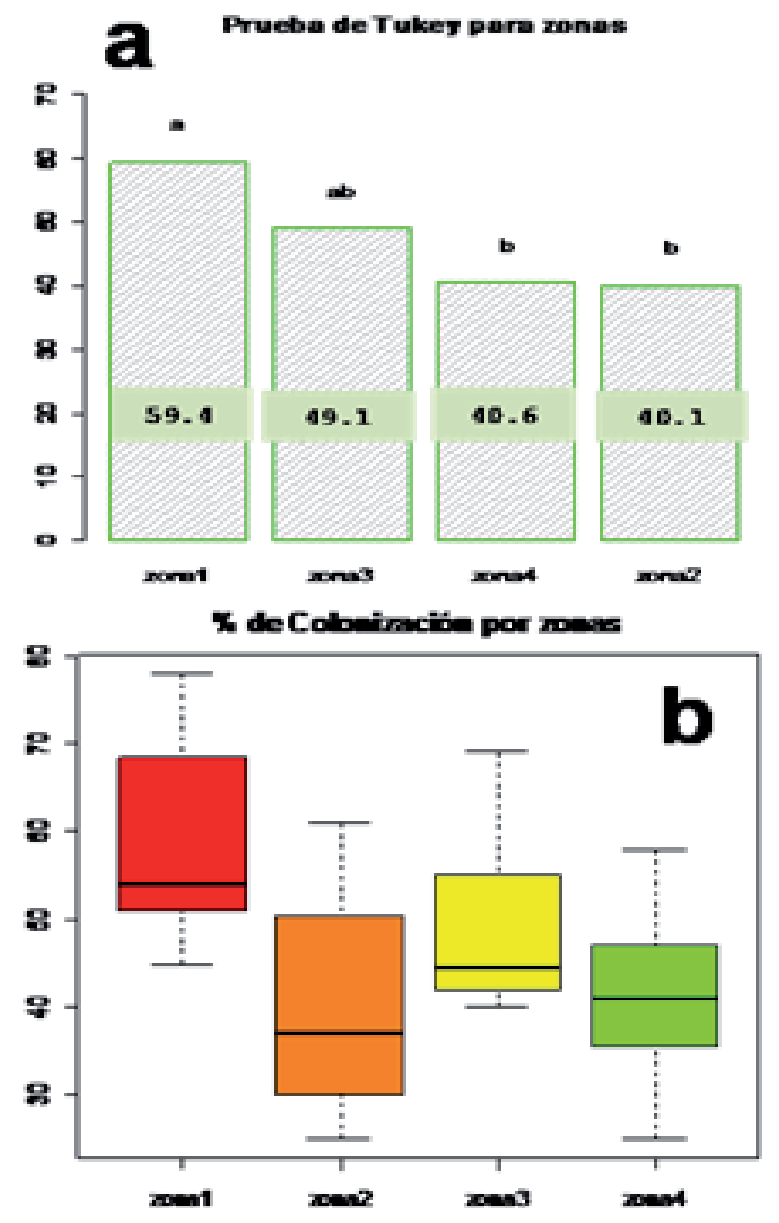

Figura 1. Resultados de la prueba de Tukey (a) y gráfico de boxplot (b) de la distribución de zonas para \% promedio de colonización HMA.
La figura 1b, muestra la variabilidad de cada una de las zonas para el porcentaje de colonización. Se cumplieron los supuestos de normalidad y homogeneidad de varianzas para el porcentaje de colonización $(p$-value $=0.178$ para la prueba de ShapiroWilk; $p$-value $=0.679$ para la prueba de Bartlett). EI ANOVA para porcentajes de colonización de micorrizas arbusculares en raíces de colosoana, señala diferencias altamente significativas ( $p$-value $=0.02359$ ) entre zonas agroecológicas. Los resultados de la prueba de Tukey, muestra que las zonas 2 y 4 son estadísticamente similares con valores promedios menores para porcentaje de colonización; mientras que, las zonas 1 y 3 presentan los mayores porcentajes de colonización (Figura 1a).

En la figura $2 b$, se observa que los hongos formadores de micorrizas arbusculares presentaron diferentes patrones de
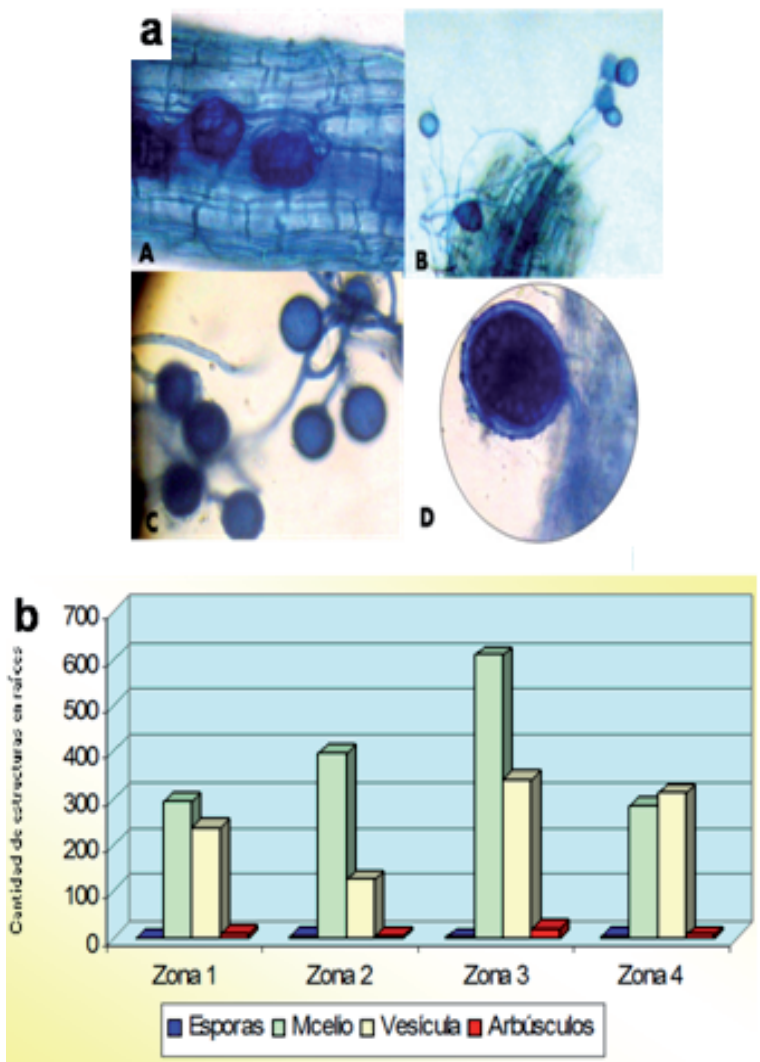

Figura 2. Modelo de colonización HMA en raíces de Bothriochloa pertusa (L) A. Camus, (a): A-vesícula; B-micelio; C y D- Esporas. (b): por zonas agroecológicas. 
colonización sobre las raíces del pasto colosuana. Las principales estructuras de colonización encontradas correspondieron a: esporas, micelios, vesículas y arbusculos. La figura muestra una mayor presencia de micelio y vesículas en la zona agroecológica 3 ; en las zonas 1 y 2 se observa mayor cantidad de micelio y en la zona 4 presencia de vesícula. En todas las zonas agroecológicas analizadas fue encontrada una menor cantidad de estructuras colonizantes correspondientes a esporas y arbusculos.

Al relacionar parámetros físico-químicos en las fincas ganaderas, mediante el análisis de componentes principales (Figura 3), la primera componente (eje " $X ")$, contrapone la

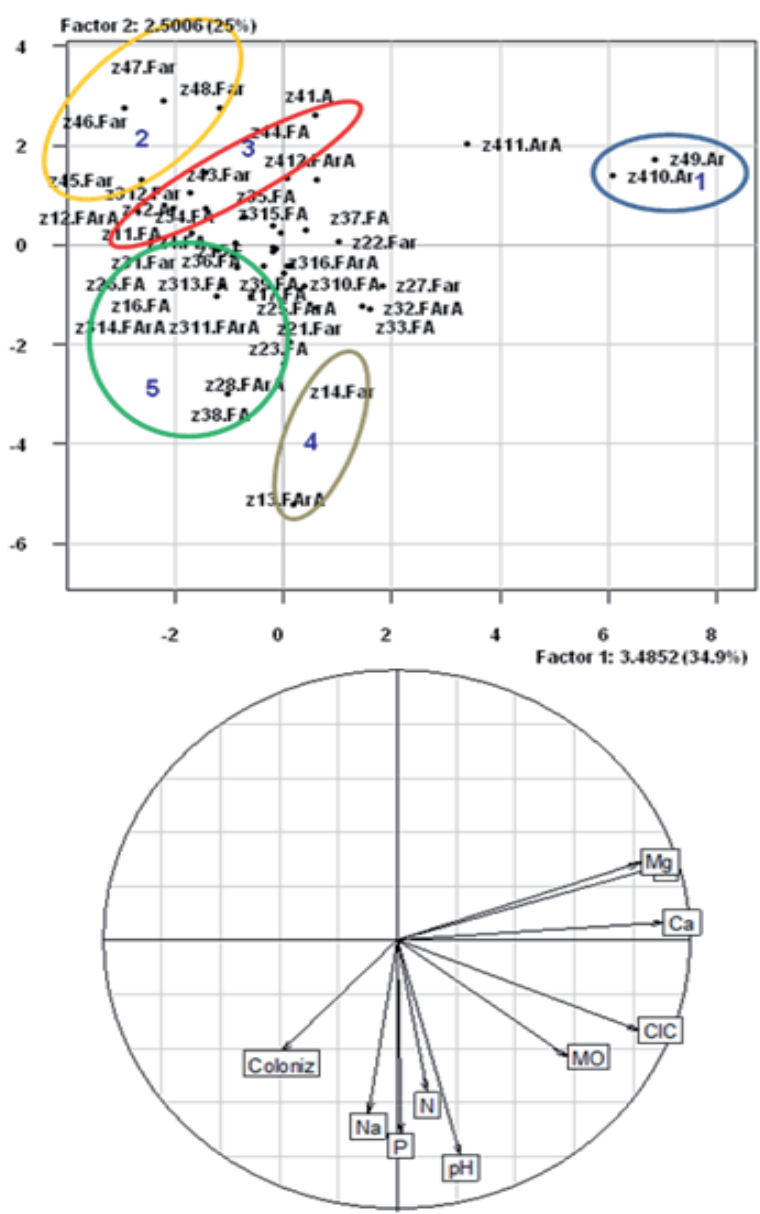

Figura 3. Análisis en componentes principales para parámetros físicoquímicos y colonización sobre las diferentes fincas en las zonas de estudio.1-5: grupos de fincas relacionadas a parámetros físicoquímicos. capacidad de intercambio catiónico (Calcio, Magnesio y Potasio) con el porcentaje de colonización ( $\ddot{e}_{1}=3.48$, explica el $35 \%$ de la variabilidad total de los datos). Las fincas 9, 10,11 y 12 de la zona 4 presentan valores alto de Magnesio (20.5 meq/100g), Calcio (32.2 meq/100g), Potasio $(1.3 \mathrm{meq} / 100 \mathrm{~g})$ y se registran los menores porcentajes de colonización.

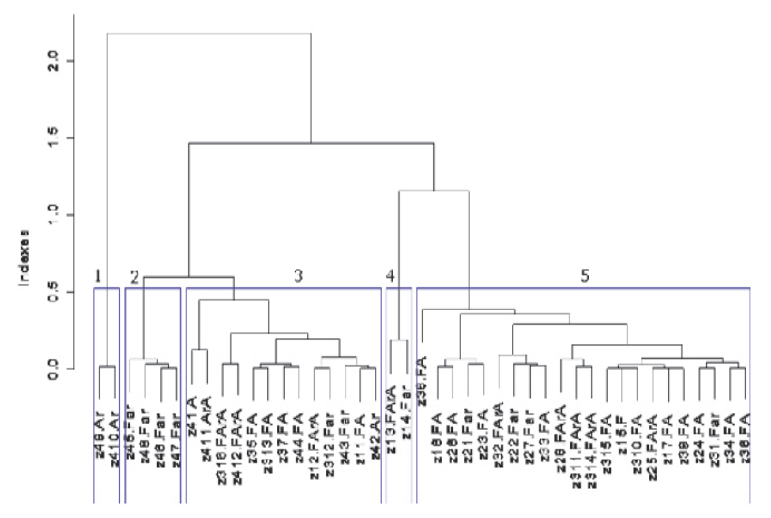

Figura 4. Análisis de clúster aglomerativo entre porcentaje de colonización y tipo de estructuras de suelos por fincas ganaderas. 1-5: grupos de fincas relacionadas a parámetros físicoquímicos.

La segunda componente (eje "Y") está relacionada a Fósforo, $\mathrm{pH}$, Nitrógeno y Sodio $\left(\ddot{e}_{2}=2.5\right.$, explica el $25 \%$ de la variabilidad total de los datos), por ejemplo las fincas de las zonas 1 y 3 , presentan altos porcentajes de colonización cuando se dan contenidos moderados de Fosforo (18.94 ppm), Nitrógeno $(0.03 \%)$, valores muy bajos de Sodio $(1.7 \mathrm{meq} / 100 \mathrm{~g})$ y $\mathrm{pH}$ medianamente alcalino (7.8).

Al analizar los porcentajes de colonización con tipos de estructuras de los suelos mediante análisis de cluster aglomerativo (Figura 4) teniendo en cuenta el análisis en componentes principales para parámetros físico-químicos (Figura 3), mostró 5 grupos de fincas relacionadas a porcentajes de colonización en forma descendente. En el grupo 1 están presentes fincas con estructura arcillosa, relacionadas con bajos porcentajes de colonización y altos contenidos de Magnesio, Calcio y Potasio; el grupo 2 está constituido por fincas con estructura franco-arenosa, con altos 
porcentaje de colonización, altos valores de capacidad de intercambio catiónico (CIC) y altos contenidos de materia orgánica. En el grupo 3, se muestran fincas con estructuras arenosa, arcillosa, franco arenosa, franco arcillosa a franco arcilloarenosa con valores medios en porcentaje de colonización, cuando además están presentes valores moderados de Magnesio, Calcio, Potasio, materia orgánica, CIC, pH, Nitrógeno, Fosforo y Sodio; en el grupo 4, se observan fincas con estructuras franco arcillosa a franco areno-arcilloso, con altos porcentajes de colonización en presencia de bajos contenidos de Sodio, Nitrógeno y pH. Finalmente en el grupo 5, se encontraron fincas con altos porcentajes de colonización en presencia de bajos contenidos de Magnesio, Calcio y Potasio, cuando están presentes estructuras de los suelos de franco arenosa y franco arcillosa.

\section{DISCUSIÓN}

En los suelos de las fincas ganaderas objeto del presente estudio en los últimos no se ha realizado ninguna práctica agrícola para mejorar las propiedades físico-químicas del suelo, principalmente su aireación, ni abonamiento para suplir la extracción de nutrientes que la especie de pasto ha venido haciendo a través del tiempo. A pesar de esta serie de limitaciones edafológicas y de manejo, el pasto colosuana, presenta algunas características deseables para el empresario ganadero como son: rápida recuperación después del pastoreo, especie altamente estolonífera, alta producción de semillas, resistencia al pisoteo y buena gustosidad para el ganado.

Estudios relacionados a la influencia de parámetros físicos-químicos sobre el establecimiento (colonización) in situ en raíces de colosuana con HMA, son escasos, lo que dificulta hacer una comparación con los resultados obtenidos en el presente trabajo. Existe evidencias de alta colonización de HMA en otras especies de pasturas como Andropogon gerardi, cuando en los suelos se presentan niveles bajos a moderados de Fosforo, Nitrógeno, Materia Orgánica y valores de $\mathrm{pH}$ medianamente alcalinos (18).
El establecimiento (colonización) de los HMA bajo condiciones de campo están determinado por diversos condiciones tales como: factores físico-químicas del suelo $(\mathrm{pH}$, contenido de fósforo, temperatura, aireación, textura y contenido de materia orgánica), condiciones climáticas (intensidad y duración de la luz, temperaturas, humedad, épocas de lluvias y épocas secas) y por las prácticas agronómicas (preparación del terreno, aplicación de pesticidas y prácticas culturales). En los suelos el efecto del $\mathrm{pH}$ es difícil de evaluar, debido a que las propiedades químicas cambian con el $\mathrm{pH}$, al igual que la solubilidad y disponibilidad de otros elementos hacia las raíces de las plantas en el suelo, incluyendo hierro, manganeso, cobre, zinc y cantidades tóxicas de aluminio. Los hongos formadores de micorrizas arbusculares tienen amplia capacidad de adaptación, se han encontrado en rangos de $\mathrm{pH}$ desde 2.7 a 9.2. Sin, embargo en suelos su formación se requiere $\mathrm{pH}$ de 4.5 a 5.5 (19-21).

Los efectos del fosforo en el suelo pueden estar asociados a otros factores como: tipo de suelo, $\mathrm{pH}$ y niveles de nitrógeno. Los altos y bajos niveles de fósforo, así como la fertilización nitrogenada reducen el porcentaje de colonización de las micorrizas, en tanto que niveles moderados de $\mathrm{P}$, incrementan los niveles de nitrógeno y la colonización de estos microorganismos. La forma del nitrógeno inorgánico en el suelo influye tanto en el porcentaje de colonización, la longitud de las raíces y la presencia estructuras colonizantes como los arbusculos y vesículas (23).

La absorción de fósforo en las especies de pastos, Digitaria ciliaris (especie pobremente colonizada por HMA), Ixeris denticulate (especie moderadamente colonizada por HMA) y Kummerowia striata (altamente colonizada por HMA), sembradas en asocios $y$ en forma individual fueron evaluadas en China con niveles limitante de fósforo. Los resultados demostraron que las pasturas altamente colonizadas con micorrizas muestran un impacto positivo sobre la coexistencia de las otras pastoras asociadas con esta en relación a patrones de 
colonización y la eficiencia en la absorción de fósforo (24). Estudios comparativos de diferentes fuentes de fósforo y la colonización de micorrizas arbusculares en raíces de trigo forrajero, demostraron que la fertilización con roca fosfórica, inducen incrementos de las micorrizas en el suelo y un alto porcentaje de colonización de esta micorrizas nativas con el trigo (25).

Diferentes investigaciones demuestran que las micorrizas arbusculares aumentan la eficiencia en la absorción de fósforo mineral $(26,27)$, a partir del fósforo solubilizado por otros microorganismos que actúan sinérgicamente $(28,29)$.

Los hongos micorrízicos arbusculares (HMA) se caracterizan por presentar un crecimiento intra e intercelular en la corteza de la raíz y por formar dos tipos de estructuras, arbúsculos y vesículas. Los arbúsculos son hifas que se dividen dicotómicamente, son invaginados por la membrana plasmática de las células corticales y presentan periodos de vida cortos, mientras que las vesículas son estructuras de almacenamiento que se forman en la parte terminal de las hifas. En raíces del pasto colosuana fueron encontradas diversas estructuras colonizantes con mayor predominio de vesículas y menor presencia de arbusculos en la condiciones del experimento. Diferentes patrones de colonización de micorrizas arbusculares como micelios, cordones hifas, vesícula y arbusculos de micorrizas arbusculares fueron observadas en células corticales de raíces en la especie de pasto Bothriochloa pertusa, y arboles leguminosas de Acacia farnesiana, Breynia fruticosa, Cyanotis cristata, Vitex negundo, Sida acuta, Polyalthia cerasoides, Boea hygrometrica (30).

La relación HMA-planta no es considerada específica, debido a que cualquier especie de HMA puede colonizar o formar simbiosis con cualquier planta, ya que se encuentran en todo tipo de suelos prácticamente. No obstante, bajo ciertas condiciones edafoclimáticas, algunos hongos pueden beneficiar mejor o en mayor grado un determinado hospedero.
El pH, la humedad del suelo y la disponibilidad de nutrientes influyen no solo en la colonización sino también en el número de esporas producidas por los hongos formadores de micorrizas arbusculares. Los HMA son encontrados en todo tipo de suelos y pueden colonizar cualquier planta que establezca simbiosis con ellos, sin embargo, las condiciones físico-químicas del suelo podrían estar generando cierto tipo de especificidad con respecto a las plantas hospederas, según las respuestas que muestran las plantas a determinadas especies de HMA.

La mayoría de los estudios existentes, relacionan la potencialidad y la eficiencia de la inoculación de HMA en diferentes cultivos en in vitro y en casa de vegetación invernadero. Pocos estudios han evidenciado el efecto que tienen las condiciones ambientales sobre el establecimiento de las HMA en diferentes ecosistemas. Este estudio se convierte en uno de los primeros a nivel del Caribe Colombiano que muestra como ciertos parámetros físico-químicos $(\mathrm{pH}$, Fosforo, Nitrógeno y sodio) de suelos de fincas ganaderos en el departamento de Sucre tienen un efecto directo sobre el establecimiento de HMA sobre raíces de colosuana. Es importante seguir evaluando in situ, los efectos de condiciones ambientalessobre la colonización de estos microorganismos en diferentes nichos ecológicos con pasturas.

Las micorrizas son un recurso biológico cuyo manejo y conservación, además de los efectos sobre la productividad vegetal, genera beneficios ambientales al mejorar las condiciones físico-químicas y biológicas del suelo. Los beneficios desde el punto de vista biológico, se derivan de su interacción con los diversos grupos de macro y microorganismos de la rizósfera, tales como aquellos implicados en el ciclaje de nutrientes (bacterias fijadoras de nitrógeno y los microorganismos solubilizadores de fosfato). Así mismo, dichos hongos interactúan con microorganismos implicados en el control biológico de patógenos presentes en el suelo, demostrando que existen diferentes tipos de interacción con las micorrizas arbusculares. 


\section{REFERENCIAS}

1. Miransari M, Bahrami HA, Rejali F, Malakouti MJ. Effects of soil compaction and arbuscular mycorrhiza on corn (Zea mays L.) nutrient uptake. Soil and Tillage Research 2009; 103(2):282-290.

2. Strullu-Derrien Ch, Strullu Désiré G. Mycorrhization of fossil and living plants. C R Palevol 2007; 6(67):483-494.

3. Corwell W, Bedford B, Chapin C. Ocurrence of arbuscular mycorrhizal fungi in a phosphorus poor wetland and mycorrhizal response to phosphorus fertilization. Am J Bot 2001; 88: 18-24.

4. Tang F, White J, Charvat I. The effect of phosphorus availability on arbuscular mycorrhizal colonization of typha angustifolia. Mycologia 2001; 93(6):1042-1047.

5. Cardoso IM, Kuyper TW. Mycorrhizas and tropical soil fertility. Agric Ecosyst Environ 2006; 116(1-2):72-84.

6. Pozo MJ., Azcón-Aguilar C. Unraveling mycorrhiza-induced resistance. Curr Opin Plant Biol 2007; 10:393-398.

7. Aguilera MM. Documento de trabajo sobre economía regional. Economía Regional: La Economía del Departamento de Sucre: Ganadería y Sector Público, Sincelejo: Banco Ganadero; 2005.

8. Totola M, Chaer G. Microrganismos e processos microbiológicos como indicadores da qualidade dos solos. Rev Bras Cienc Solo 2002; 2:249-259.

9. Barea J. Rhizosphere and mycorrhizal of frield crops: Biological resource management connecting. Connecting Science and Policy. (Balázs E, Galante E, Lynch JM, Schepers JS, Toutant JP, Werner D, Werry PA Th J, eds) Berlin, Heidelberg, New York: INRA Editions, Springer-Verlag; 2002.
10. Plan de Ordenamiento Territorial. Diagnóstico del Municipio de Sincelejo. Sincelejo: Plan de Ordenamiento Territorial; 2002.

11. Instituto Geográfico Agustín Codazzi. Estudio general de suelos y zonificación de tierras. Departamento de Sucre. Sincelejo: Instituto Geográfico Agustín Codazzi; 1998.

12. Pérez Cordero A. Eficiencia de hongos formadores de micorrizas arbusculares (H.M.A.) nativos, asociados a la producción de forraje en la especie de pasto colosuana (Bothriochloa pertusa (L) A. camus) en el municipio de Corozal; departamento de Sucre. [Tesis de Maestría]. Bogotá, Colombia: Universidad de los Andes, Facultad de Ciencias, Departamento de Ciencias Biológicas, 2003.

13. Vertel Morrinson M. Diseño y Análisis de Experimentos en Ciencias Agroindustriales. Sincelejo: Universidad de Sucre; 2005.

14. Lebart L, Morineau A., Piron M. Statisitique exploratoire multidimensionnelle. Paris: Dunod; 1995.

15. Cabarcas G., Pardo CE. Métodos estadísticos multivariados. Simposio de estadística en línea 2001, URL disponible en: http://www.docentes.unal.edu.co/ cepardot/docs/imposioestadistica.

16. Pardo CE, Del-Campo PC. Combinación de métodos factoriales y de análisis de conglomerados en $\mathrm{R}$ : el paquete FactoClass', Revista Colombiana de Estadística en línea 2007; 30(2) disponible en:http://www.matematicas. unal.edu.co/revcoles/.

17. R development core team R: A Language and Environment for Statistical computing, R Foundation for Statistical Computing, en línea 2009, disponible en:http://www.r-project.org. 
18. Barea J., Jefries P. Arbuscular mycorrhyzal in sustainable soil-plant systems. En: Varma, A. y B. Hock (eds.). Mycorrhiza: structure, function, molecular biology and biotechnology. Berlin: Springer-Verlag; 1995.

19. Sánchez de Prager M. Endomicorrizas en agroecosistemas colombianos, ed., Bogotá: Universidad Nacional de Colombia, sede Palmira; 1999.

20. Peña L. Las micorrizas vesícula arbusculares (MA), una alternativa sostenible para los sistemas de producción de la zona andina de Nariño. Boletin técnico 9. Corpoica; 1999.

21. Alloush GA, Zeto SK, Clark RB. Phosphorus source organic matter and arbuscular mycorrhizal effects on growth and mineral acquisition of chickepea grow acidic soil. J Plant nutr 2000; 23(9):1351-1369.

22. A.J. V, Orborne BA, DT M. Form de nitrogen inorganic influence mycorrhizal colonisation and photosynthesis of cucumber. Sci Hortic 2002; 92(34):229-239.

23. Xin $\mathrm{CH}$, Jianjun $\mathrm{T}$, Guiye $\mathrm{Z}$, Shuijin $\mathrm{H}$. Arbuscular mycorrhizal colonization and phosphorus acquisition of plants: effects of coexisting plant species. Appl Soil Ecol 2005; 28(3):259-269.

24. Covacevich F, Marino MA, Echeverría HE. The phosphorus source determines the arbuscular mycorrhizal potential and the native mycorrhizal colonization of tall fescue and wheatgrass. Eur J Soil Biol 2006; 42(3):127-138.
25. Cui M, Caldwell MW. Shading reduces exploitation of soil nitrate and phosphate by Agropyron desertorum and Artemisia tridentata from soils with patchy and uniform nutrient distributions. Oecologia 1997; 109(2):177-183.

26. Osorio NW., Habte M. Synergistic influence of an arbuscular mycorrhizal fungus and $a$ P solubilizing fungus on growth and $\mathrm{P}$ uptake of Leucaena leucocephala in an Oxisol. Arid Land Research and Management 2001; 15(3):263-274.

27. Villegas J., Fortin JA. Phosphorus solubilization and $\mathrm{pH}$ changes as a result of the interactions between soil bacteria and arbuscular mycorrhizal fungi on a medium containing $\mathrm{NH}_{4}{ }^{+}$ as nitrogen source. Can J Bot 2001; 79:865-870.

28. Singh S, Kapoor KK. Effects of inoculation of phosphate-solubilizing microorganisms and arbuscular mycorrhizal fungus on mung bean grown under natural soil conditions. Mycologia 1998; 7(5):249-253.

29. Ramírez A., Otalvaro D., Álvarez C., Pérez J.C., Osorio NW. Efectos de organismos rizosféricos sobre la absorción de fosfato y el crecimiento de Leucaena en un Andisol. Suelos Ecuatoriales 2001; 31(2):239-243.

30. Tao L., Zhiwei Z. Arbuscular mycorrhizas in a hot and arid ecosystem in southwest China. Appl Soil Ecol 2005; 29(2): 135-141. 